\title{
ANALYSIS OF COMBINING ABILITY, GENE ACTION AND HETEROSIS IN A FULL DIALLEL CROSS OF BREAD WHEAT
}

\author{
Ismail Hussain Ali
}

College of Agric. Salahaddin Univ.
Fahmi Salih Sulaiman

Agriculture Research Station/Koya

Email: dr.ismail@ymail.com

\section{ABSTRACT}

This experiment involved implementation of a full diallel crosses between five genotypes of bread wheat in the 2009-2010. Then the parents and their $F_{1}$ generation including reciprocals were cultivated in the 2010-2011 growing season at the Agricultural Research Station in Koya / Erbil under rainfall conditions using a randomized complete block design with four replications. Some growth characters, grain yield and its components were studied. Genetic statistical analysis were done to estimate general combining ability (GCA) of the parents and specific combining ability (SCA) of $F_{1}$ hybrids and reciprocals, also the genetic variance components (additive and dominance), environmental variance, average degree of dominance, heritability in broad and narrow sense, expected genetic advance, heterosis and genetic correlation coefficients among the characters were estimated. The results showed that some parents exhibited positive and high GCA, while some hybrids showed SCA for the majority of the characters. The values of additive genetic variance was more than the values of dominant genetic variance for days to flowering, days to maturity, plant height, spike length, 1000-grain weight, and harvest index. While the values of dominance genetic variance was more than additive for flag leaf area, number of spikes per plant, number of grains per spike, biological and grain yield per plant. The values of heritability in broad sense were high for all characters, while heritability in narrow sense was high for some characters but moderate for others. The values of average degree of dominance was greater than one for flag leaf area, number of spikes per plant, number of grains per spike, biological and grain yield per plant. The expected genetic advance was moderate for plant height, spike length, and 1000-grain weight. Grain yield per plant revealed a significant positive correlation with flag leaf area (0.921), number of spikes per plant (0.883) and 1000 grain weight (0.698).

Keywords: Bread wheat, Diallel cross, Combining ability, Gene action, Heterosis, Received 5/12/2011 Accepted 10/9/2012.

\section{INTRODUCTION}

In plant breeding programs, diallel crosses are mostly used to provide information on genetic effects for a number of parental varieties or estimates of general and specific combining ability variance components and heritability for plant population from randomly chosen parental varieties. This technique starts to develop with the developing concept of general and specific combining ability by Sprague and Tatum (1942), then Jinks and Hayman (1953), Hayman (1954), Griffing (1956) and Kempthorne (1969), presented various statistical analysis for diallel crossing. Combining ability analysis is a biometrical method which identifies the parent varieties possessing best combining ability and genetic effects involved in the parent varieties possessing best combining ability and genetic effects

A part of M. Sc. thesis of the second author. 
involved in inheritance of various plant characters (Griffing, 1956). It also helps to select the cross combinations having desirable characters to enhance grain yield in wheat and to determine the nature and extent of various types of genetic effects involved in the expression of polygenic character. These points are helpful for selecting parents for next hybridization program. General and specific combining ability had been studied for the different characters in wheat by many researchers using $F_{1}$ generation. Kashif and Khaliq (2003) reported that mean square of G.C.A. were significant for plant height, flag leaf area, spikes per plant, spike length and grains per spike, Mean squares of S.C.A. were significant for all characters except spikes per plant, whereas the reciprocal mean square were significant for spike length and grain yield per plant only. Many researchers worked on wheat and observed the presence of additive type of gene action for grain yield per plant and some characters, but some other researchers determined involvement of dominance for these characters. Selection would be successful during the early generations for characters that controlled by additive gene action; otherwise, the selection would be effective at later generations (Liu, 2005). Additive gene action was observed for flag leaf area, spikes per plant, spike length, grains per spike, while dominance gene action was observed for plant height, 1000 grain weight and grain yield per plant (Kashif and Khaliq, 2003). Akram et al. (2009) reported that spike length, grains per spike, 1000-grain weight and grain yield per plant were controlled by dominance gene action, and average degree of dominance was greater than one, it means that these characters controlled by over dominance. On the other hand, heritability estimates indicate that certain morphological characters that influence grain yield in wheat are more heritable than yield because yield is a polygenic character and is greatly influenced by the environment. So, selection of wheat varieties on the basis of yield is difficult and needs to be delayed. High heritable estimates for spikes per plant, spike length, and1000-grain weight found by Cheema et al. (2006) in their studies of full diallel crosses between five varieties of wheat. Many workers worked on hybrid wheat and found that most of $\mathrm{F}_{1}$ hybrids and reciprocals showed significant and desirable hrterosis for spikes per plant, spike length, grains per spike and grain yield per plant (Ansari, 2002), while Ilker et al. (2010) observed that all hybrids showed significant and positive heterosis over mid parents for spike length and fourteen hybrids for grains per spike and two hybrids for 1000-grain weight.

The present investigation is employed to study the magnitude of combining ability effects of parents and crosses, gene action controlling the inheritance, estimation of variability parameters, heritability, genetic advance and genetic correlation coefficients of growth characters, yield and its components in bread wheat (Triticum aestivum L.).

\section{MATERIALS AND METHODS}

Five bread wheat genotypes (Triticum aestivum L.) which their numbers and pedigrees are presented in Table (1) obtained from Sulaymania and Erbil Research Center were crossed in a complete diallel during 2009-2010. The $F_{1}$ grains and reciprocals and their parents were sown during the next growing season 2010-2011 at the Agricultural Research Station in Koya / Erbil [under rainfall conditions (487 $\mathrm{mm}$ ) depend on meteorological in the search site] using a randomized complete block design with four replications. Each replication consist 25 rows (in addition 2 
rows as guard), one row for each genotype, $3.0 \mathrm{~m}$ long, with $20 \mathrm{~cm}$ row to row and $15 \mathrm{~cm}$ plant to plant distance. The data were recorded from mean of ten plants randomly selected in each row for days to flowering, days to maturity, plant height, flag leaf area, spike length, spikes per plant, grains per spike, 1000-grain weight, biological yield, grain yield per plant and harvest index. Mean values of 10 plants from each replication for all the characters were subjected to analysis of variance technique and the significant differences among the means were compared according to least significant difference (LSD) test. Estimates of combining ability were computed by using the method as described by Griffing (1956) Method I, random model. The G.C.A. effects for the parents, S.C.A. effects for the $F_{1}$ hybrids and reciprocal effects were calculated. Also estimations of variance for general and specific combining ability effects of the parents, genetic components variance (Additive and dominance), environment variance, heritability in broad and narrow senses, average degree of dominance, expected genetic advance . Each of heritability in broad sense, narrow sense, and genetic advance was categorized as low, moderate and high as suggested by Ali (1999), Adary (1987) and (Agarwal and Ahmad, 1982), respectively. Heterosis over mid parents was computed according to Singh and Chaudhary (1985) and genetic correlation between studied characters was calculated according to Walter (1975).

Table (1): Pedigree of used genotypes as parents and their sources .

\begin{tabular}{|c|l|l|}
\hline No & \multicolumn{1}{|c|}{ Source } & \multicolumn{1}{c|}{ Pedigree } \\
\hline 1. & $\begin{array}{l}\text { A.R.C. } \\
\text { Erbil }\end{array}$ & Saberbeg X UP 114 ( Adnania) \\
\hline 2. & $\begin{array}{l}\text { A.R.C. } \\
\text { Sulaymai }\end{array}$ & PBW 450 - ONPL \\
\hline 3. & $\begin{array}{l}\text { A.R.C. } \\
\text { Sulaymai }\end{array}$ & BHRIKUTI NL623 - ONPL \\
\hline 4. & $\begin{array}{l}\text { A.R.C. } \\
\text { Sulaymai }\end{array}$ & $\begin{array}{l}\text { PASTOR/3/KAUZX2/OPATA/KAUZCMSS93 B003085-29Y- } \\
\text { 010M-010Y-010M-7Y-OM }\end{array}$ \\
\hline 5. & $\begin{array}{l}\text { A.R.C. } \\
\text { Sulaymai }\end{array}$ & $\begin{array}{l}\text { KAUZ//ALTRA84AOS/3/MILAN/KAUZ/4/HUITES } \\
\text { CMSS97-M03912T-040Y-020Y-030M-020Y-040M-LY-2M-OY }\end{array}$ \\
\hline
\end{tabular}

\section{RESULTS AND DISCUSSION}

The analysis of variance revealed significant genotypic differences for all the characters studied (Table 2). The means of the parents and crosses for the characters (Table, 3) had revealed that parent (1) exceeded over the other parents in most characters, it was the earliest in days to flowering, and better in plant height, flag leaf area, spike length, spikes per plant, biological yield per plant and grain yield per plant. Parent (2) exceeded for spikes per plant and grains per spike and parent (4) for 1000-grain weight and harvest index. Regarding the crosses, it was noted that hybrid $(5 \times 1)$ exceeded for most characters earlier to flowering, flag leaf area, spikes per plant, 1000- grain weight, biological yield and grain yield per plant. Hybrid $(1 \times 4)$ exceeded for plant height and spike length. Hybrid $(2 \times 4)$ was the earliest to maturity. Hybrid $(5 \times 2)$ had the highest number of grains per spike, and the hybrid $(3 \times 5)$ exceeded for harvest index. Combining ability analysis (Table 4$)$ showed that means squares for GCA and SCA were highly significant for all the characters except days to maturity which was not significant for SCA, whereas, 
Mesopotamia J. of Agric.

Vol. (42) No. (1) 2014
ISSN: 2224-9796 (Online) ISSN: $1815-316 \mathrm{X}$ (Print)

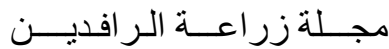

المجلد(42) العدد(1) 2014 اعنرأ

mean squares for reciprocals were significant for plant height, flag leaf area, spike length, grains per spike and biological yield per plant.

Table (2): Analysis of variance for the characters studies in a $5 \times 5$ diallel cross of bread wheat.

\begin{tabular}{|l|c|c|c|c|c|c|c|}
\hline SOV & df & $\begin{array}{c}\text { Days to } \\
\text { flowering }\end{array}$ & $\begin{array}{c}\text { Days to } \\
\text { maturity }\end{array}$ & $\begin{array}{c}\text { Plant height } \\
(\mathrm{cm})\end{array}$ & $\begin{array}{c}\text { Flag leaf } \\
\text { area }\left(\mathrm{cm}^{2}\right)\end{array}$ & $\begin{array}{c}\text { No. of } \\
\text { spikes/plant }\end{array}$ & $\begin{array}{c}\text { Spike } \\
\text { length } \\
(\mathrm{cm})\end{array}$ \\
\hline Replications & 3 & 2.4 & 1.573 & 2.160 & 81.245 & 0.892 & 2.298 \\
Genotypes & 24 & $18.769^{* *}$ & $7.294^{* *}$ & $\begin{array}{c}207.588^{* *} \\
102.310^{* *}\end{array}$ & $8.556^{* *}$ & $3.558^{* *}$ \\
Error & 72 & 1.712 & 3.011 & 5.986 & 9.117 & 1.328 & 0.130 \\
\hline
\end{tabular}

\begin{tabular}{|l|c|c|c|c|c|c|}
\hline SOV & df & $\begin{array}{c}\text { No. of } \\
\text { grains/spike }\end{array}$ & $\begin{array}{c}\text { 1000-grain } \\
\text { weight (g) }\end{array}$ & $\begin{array}{c}\text { Biological } \\
\text { yield/plant (g) }\end{array}$ & $\begin{array}{c}\text { Grain } \\
\text { yield/plant (g) }\end{array}$ & $\begin{array}{c}\text { Harvest } \\
\text { index (\%) }\end{array}$ \\
\hline Replications & 3 & 147.509 & 7.695 & 231.126 & 44.007 & 5.710 \\
Genotypes & 24 & $57.645^{* *}$ & $64.393^{* *}$ & $648.686^{* *}$ & $104.905^{* *}$ & 10.410 \\
Error & 72 & 12.850 & 5.662 & 82.806 & 19.599 & 2.313 \\
\hline
\end{tabular}

** Significant $(\mathrm{P}=0.01)$

Table (3): Mean performance of parents and hybrids for the studied characters.

\begin{tabular}{|c|c|c|c|c|c|c|}
\hline & $\begin{array}{l}\text { Days to } \\
\text { flowering }\end{array}$ & $\begin{array}{l}\text { Days to } \\
\text { maturity }\end{array}$ & $\begin{array}{c}\text { Plant } \\
\text { height }(\mathrm{cm})\end{array}$ & $\begin{array}{c}\text { Flag leaf } \\
\text { area }\left(\mathrm{cm}^{2}\right)\end{array}$ & $\begin{array}{c}\text { No. of } \\
\text { spikes/plant }\end{array}$ & $\begin{array}{l}\text { Spike } \\
\text { length } \\
(\mathrm{cm})\end{array}$ \\
\hline \multicolumn{7}{|l|}{ Parents } \\
\hline 1 & 128.25 & 172.5 & 119.67 & 53.78 & 11.75 & 15.33 \\
\hline 2 & 132.00 & 170.75 & 95.24 & 43.91 & 11.75 & 11.94 \\
\hline 3 & 134.75 & 172.25 & 98.51 & 45.09 & 8.90 & 13.09 \\
\hline 4 & 136.50 & 173.75 & 101.65 & 46.41 & 9.05 & 13.25 \\
\hline 5 & 129.25 & 172.00 & 98.61 & 42.02 & 10.40 & 12.34 \\
\hline \multicolumn{7}{|l|}{ F1 hybrids } \\
\hline $1 \times 2$ & 129.50 & 173.00 & 115.36 & 58.99 & 14.40 & 14.30 \\
\hline $1 \times 3$ & 130.25 & 172.75 & 111.78 & 55.59 & 12.05 & 14.27 \\
\hline $1 \times 4$ & 131.50 & 174.50 & 117.96 & 58.53 & 13.78 & 15.15 \\
\hline $1 \times 5$ & 129.75 & 174.25 & 113.51 & 56.59 & 11.93 & 14.25 \\
\hline $2 \times 3$ & 133.50 & 172.25 & 100.41 & 52.35 & 11.50 & 12.78 \\
\hline $2 \times 4$ & 130.75 & 170.50 & 101.00 & 49.05 & 11.60 & 12.64 \\
\hline $2 \times 5$ & 130.50 & 171.25 & 102.05 & 47.59 & 11.93 & 12.45 \\
\hline $3 \times 4$ & 136.50 & 174.00 & 102.80 & 45.99 & 10.00 & 13.08 \\
\hline $3 \times 5$ & 130.75 & 171.50 & 103.69 & 48.54 & 11.75 & 13.28 \\
\hline $4 \times 5$ & 131.00 & 173.75 & 107.13 & 52.96 & 11.80 & 13.46 \\
\hline \multicolumn{7}{|l|}{$\underline{\text { Reciprocals }}$} \\
\hline $5 \times 4$ & 130.50 & 171.50 & 105.05 & 53.60 & 11.95 & 13.88 \\
\hline $5 \times 3$ & 130.50 & 172.00 & 103.93 & 53.18 & 12.25 & 13.28 \\
\hline $5 \times 2$ & 130.50 & 171.25 & 104.15 & 53.12 & 12.35 & 13.15 \\
\hline $5 \times 1$ & 129.50 & 174.75 & 115.86 & 61.58 & 14.88 & 15.00 \\
\hline $4 \times 3$ & 134.50 & 173.75 & 101.08 & 52.99 & 10.90 & 13.36 \\
\hline $4 \times 2$ & 131.25 & 172.75 & 106.05 & 52.02 & 12.03 & 13.16 \\
\hline $4 \times 1$ & 131.25 & 174.50 & 116.96 & 55.70 & 13.18 & 15.06 \\
\hline $3 \times 2$ & 133.00 & 172.25 & 99.86 & 51.35 & 11.23 & 12.55 \\
\hline $3 \times 1$ & 132.25 & 175.50 & 114.45 & 56.44 & 12.58 & 14.20 \\
\hline $2 \times 1$ & 130.00 & 174.25 & 113.95 & 57.74 & 14.25 & 14.18 \\
\hline Grand mean & 131.52 & 172.88 & 106.85 & 52.20 & 11.93 & 13.58 \\
\hline L.S.D 1\% & 2.461 & 3.264 & 4.602 & 5.679 & 2.168 & 0.679 \\
\hline
\end{tabular}


Mesopotamia J. of Agric.

Vol. (42) No. (1) 2014
ISSN: 2224-9796 (Online) ISSN: $1815-316 \mathrm{X}$ (Print)

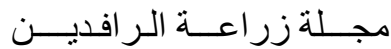

المجلد(42) العدد(1) 2014

\begin{tabular}{|c|c|c|c|c|c|}
\hline & $\begin{array}{c}\text { No. of } \\
\text { grains/spike }\end{array}$ & $\begin{array}{l}\text { 1000-grain } \\
\text { weight }(\mathrm{g})\end{array}$ & $\begin{array}{c}\text { Biological } \\
\text { yield/plant }(\mathrm{g})\end{array}$ & $\begin{array}{c}\text { Grain } \\
\text { yield/plant (g) }\end{array}$ & $\begin{array}{c}\text { Harvest } \\
\text { index }(\%)\end{array}$ \\
\hline $\begin{array}{l}\text { Parents } \\
1 \\
2 \\
3 \\
4 \\
5\end{array}$ & $\begin{array}{l}65.93 \\
73.68 \\
70.95 \\
64.18 \\
68.93\end{array}$ & $\begin{array}{l}44.82 \\
36.70 \\
48.00 \\
48.25 \\
47.05\end{array}$ & $\begin{array}{l}74.45 \\
62.83 \\
68.13 \\
56.60 \\
63.03\end{array}$ & $\begin{array}{l}30.09 \\
27.52 \\
28.15 \\
25.23 \\
27.77\end{array}$ & $\begin{array}{l}40.31 \\
43.83 \\
44.13 \\
44.51 \\
41.37\end{array}$ \\
\hline $\begin{array}{l}\text { F1 hybrids } \\
1 \times 2 \\
1 \times 3 \\
1 \times 4 \\
1 \times 5 \\
2 \times 3 \\
2 \times 4 \\
2 \times 5 \\
3 \times 4 \\
3 \times 5 \\
4 \times 5\end{array}$ & $\begin{array}{l}68.70 \\
70.20 \\
71.63 \\
60.83 \\
73.20 \\
67.25 \\
71.58 \\
68.90 \\
73.45 \\
73.73\end{array}$ & $\begin{array}{l}48.69 \\
48.14 \\
51.23 \\
53.25 \\
41.57 \\
44.18 \\
54.63 \\
46.06 \\
51.32 \\
49.93\end{array}$ & $\begin{array}{l}95.10 \\
81.58 \\
95.05 \\
86.40 \\
68.65 \\
70.03 \\
76.68 \\
64.35 \\
81.80 \\
82.05\end{array}$ & $\begin{array}{l}39.79 \\
35.61 \\
42.70 \\
33.92 \\
29.97 \\
31.55 \\
33.53 \\
28.51 \\
36.98 \\
36.67\end{array}$ & $\begin{array}{l}41.85 \\
43.56 \\
44.84 \\
39.24 \\
43.66 \\
44.98 \\
43.75 \\
44.28 \\
45.23 \\
44.72\end{array}$ \\
\hline $\begin{array}{l}\text { Reciprocals } \\
5 \times 4 \\
5 \times 3 \\
5 \times 2 \\
5 \times 1 \\
4 \times 3 \\
4 \times 2 \\
4 \times 1 \\
3 \times 2 \\
3 \times 1 \\
2 \times 1\end{array}$ & $\begin{array}{l}75.98 \\
71.60 \\
78.68 \\
67.88 \\
74.53 \\
71.70 \\
70.63 \\
70.00 \\
67.53 \\
69.18\end{array}$ & $\begin{array}{l}49.72 \\
48.78 \\
42.05 \\
54.11 \\
45.02 \\
44.08 \\
50.17 \\
41.05 \\
47.93 \\
46.78\end{array}$ & $\begin{array}{c}84.23 \\
85.75 \\
79.00 \\
110.25 \\
71.05 \\
79.08 \\
92.73 \\
66.35 \\
85.40 \\
94.15\end{array}$ & $\begin{array}{l}38.05 \\
37.31 \\
33.81 \\
44.31 \\
30.71 \\
34.44 \\
39.59 \\
28.76 \\
35.83 \\
39.93\end{array}$ & $\begin{array}{l}45.13 \\
43.48 \\
42.69 \\
40.18 \\
43.20 \\
43.55 \\
42.64 \\
43.09 \\
41.82 \\
42.34\end{array}$ \\
\hline Grand mean & 70.43 & 46.98 & 78.99 & 34.03 & 43.13 \\
\hline L.S.D $1 \%$ & 6.742 & 4.476 & 17.116 & 8.327 & 2.861 \\
\hline
\end{tabular}

Parent (1) showed desirable GCA effects for days to flowering, flag leaf area, spikes per plant, spike length, 1000-grain weight, biological yield and grain yield per plant, while parent (2) had desirable effects for days to flowering, days to maturity, plant height, spikes per plant, grains per spike and harvest index and parent (5) for days to flowering, days to maturity, plant height, 1000-grain weight, biological yield and grain yield per plant, then parent (4) for plant height, spike length, grains per spike, 1000-grain weight and harvest index and parent (3) for plant height, grains per spike and harvest index (Table, 5). Other researchers also obtained parents which showed desirable GCA effects for different mentioned characters (Akram et al., 2011, and Yadav and Sirohi, 2011). 
Mesopotamia J. of Agric. Vol. (42) No. (1) 2014
ISSN: 2224-9796 (Online) ISSN: $1815-316 \mathrm{X}$ (Print)

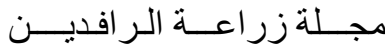

المجلد(42) العدد(1) 2014 اعلمن

Table (4): Combining ability analysis in a $5 \times 5$ diallel cross of bread wheat according to (Griffing, 1956) Method I, random model.

\begin{tabular}{|l|c|c|c|c|c|c|c|}
\hline SOV & df & $\begin{array}{c}\text { Days to } \\
\text { flowering }\end{array}$ & $\begin{array}{c}\text { Days to } \\
\text { maturity }\end{array}$ & $\begin{array}{c}\text { Plant height } \\
(\mathrm{cm})\end{array}$ & $\begin{array}{c}\text { Flag leaf } \\
\text { area }\left(\mathrm{cm}^{2}\right)\end{array}$ & $\begin{array}{c}\text { No. of } \\
\text { spikes/ } \\
\text { plant }\end{array}$ & $\begin{array}{c}\text { Spike } \\
\text { length } \\
(\mathrm{cm})\end{array}$ \\
\hline GCA & 4 & $21.923^{* *}$ & $5.317^{* *}$ & $271.637^{* *}$ & $68.761^{* *}$ & $6.486^{* *}$ & $4.656^{* *}$ \\
SCA & 10 & $2.033^{* *}$ & $1.247^{* *}$ & $13.320^{* *}$ & $26.552^{* *}$ & $1.995^{* *}$ & $0.189^{* *}$ \\
Reciprocals & 10 & $0.459^{\text {n.s. }}$ & $1.003^{\text {n.s. }}$ & $2.578^{*}$ & $7.330^{* *}$ & $0.544^{* *}$ & $0.083^{* * 0}$ \\
Error & 72 & 0.428 & 0.753 & 1.496 & 2.279 & 0.332 & .033 \\
$\sigma_{\text {gca } /}^{2} \sigma_{\text {sca }}^{2}$ & & 2.000 & 1.392 & 3.678 & 0.300 & 0.462 & 4.797 \\
\hline
\end{tabular}

\begin{tabular}{|l|c|c|c|c|c|c|}
\hline SOV & df & $\begin{array}{c}\text { No. of } \\
\text { grains/spike }\end{array}$ & $\begin{array}{c}\text { 1000-grain } \\
\text { weight }(\mathrm{g})\end{array}$ & $\begin{array}{c}\text { Biological } \\
\text { yield/plant } \\
(\mathrm{g})\end{array}$ & $\begin{array}{c}\text { Grain } \\
\text { yield/plant } \\
(\mathrm{g})\end{array}$ & $\begin{array}{c}\text { Harvest } \\
\text { index }(\%)\end{array}$ \\
\hline GCA & 4 & $24.111^{* *}$ & $68.153^{* *}$ & $428.869^{* *}$ & $44.792^{* *}$ & $9.360^{* *}$ \\
SCA & 10 & $16.010^{* *}$ & $10.067^{* *}$ & $180.285^{* *}$ & $38.312^{* *}$ & $1.658^{* *}$ \\
Reciprocals & 10 & $8.933^{* *}$ & $1.308^{\text {n.s. }}$ & $37.379^{*}$ & $6.715^{\text {n. s. }}$ & $0.844^{\text {n. s. }}$ \\
Error & 72 & 3.212 & 1.416 & 20.702 & 4.900 & 0.578 \\
$\sigma_{\text {gca }}^{2} \sigma_{\text {sca }}^{2}$ & \multicolumn{2}{|l}{0.114} & 1.136 & 0.270 & 0.041 & 1.207 \\
\hline * Significant $(\mathrm{P}=0.05)$
\end{tabular}

Table (5): Estimates of GCA effects for studied characters in a $5 \times 5$ diallel cross of bread wheat.

\begin{tabular}{|l|c|c|c|c|c|c|}
\hline parents & $\begin{array}{c}\text { Days to } \\
\text { flowering }\end{array}$ & $\begin{array}{c}\text { Days to } \\
\text { maturity }\end{array}$ & $\begin{array}{c}\text { Plant height } \\
(\mathrm{cm})\end{array}$ & $\begin{array}{c}\text { Flag leaf } \\
\text { area }\left(\mathrm{cm}^{2}\right)\end{array}$ & $\begin{array}{c}\text { No. of } \\
\text { spikes/ } \\
\text { plant }\end{array}$ & $\begin{array}{c}\text { Spike length } \\
(\mathrm{cm})\end{array}$ \\
\hline 1 & -1.470 & 0.970 & 9.071 & 4.668 & 1.127 & 1.131 \\
2 & -0.220 & -0.930 & -3.515 & -1.202 & 0.351 & -0.669 \\
3 & 1.555 & -0.030 & -3.346 & -1.544 & -0.921 & -0.280 \\
4 & 1.505 & 0.395 & -0.668 & -0.838 & -0.593 & 0.052 \\
5 & -1.370 & -0.405 & -1.542 & -1.084 & 0.036 & -0.235 \\
\hline S.E. $\left(g_{i}-g_{j}^{\prime}\right)$ & 0.131 & 0.174 & 0.245 & 0.302 & 0.115 & 0.036 \\
\hline
\end{tabular}

\begin{tabular}{|l|c|c|c|c|c|}
\hline parents & $\begin{array}{c}\text { No. of } \\
\text { grains/spike }\end{array}$ & $\begin{array}{c}\text { 1000-grain } \\
\text { weight }(\mathrm{g})\end{array}$ & $\begin{array}{c}\text { Biological } \\
\text { yield/plant }(\mathrm{g})\end{array}$ & $\begin{array}{c}\text { Grain } \\
\text { yield/plant }(\mathrm{g})\end{array}$ & $\begin{array}{c}\text { Harvest index } \\
(\%)\end{array}$ \\
\hline 1 & -2.591 & 2.014 & 9.968 & 3.156 & -1.426 \\
2 & 1.331 & -4.237 & -3.519 & -1.348 & 0.221 \\
3 & 0.699 & -0.585 & -5.889 & -2.107 & 0.525 \\
4 & 0.786 & 0.710 & -3.812 & -0.761 & 1.100 \\
5 & -0.226 & 2.098 & 3.253 & 1.061 & -0.420 \\
\hline S.E. $\left(g_{i}-g_{j}\right)$ & 0.358 & 0.238 & 0.910 & 0.443 & 0.152 \\
\hline
\end{tabular}

The data regarding SCA effects presented in Table (6) showed that the cross $(3 \times 5)$ had the desirable SCA effects for all the studied characters except plant height, followed by cross $(1 \times 4)$ which showed desirable SCA effects for all characters except days to flowering, days to maturity and plan height also a cross $(4 \times 5)$ showed desirable SCA effects for all characters except days to maturity, plant height and 1000-grain weight. They followed by cross $(1 \times 2)$ for six characters flag leaf area, spikes per plant, spike length, 1000-grain weight, biological and grain yield per plant. Then a cross $(1 \times 5)$ for five characters, flag leaf area, spikes per plant, spike length, 1000-grain weight and biological yield, and a cross $(2 \times 4)$ for 
Mesopotamia J. of Agric. Vol. (42) No. (1) 2014
ISSN: 2224-9796 (Online) ISSN: $1815-316 \mathrm{X}$ (Print)

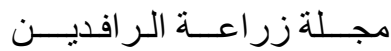

المجلد(42) العدد(1) 2014 ألمن

four characters, days to flowering, days to maturity, 1000-grain weight and biological yield, then cross $(2 \times 5)$ for spike length and number of grains per spike, and a cross $(1 \times 3)$ for days to flowering and harvest index, then cross $(3 \times 4)$ for plant height. Finally, cross $(2 \times 3)$ for flag leaf area. Other researchers also obtained desirable or non desirable SCA effects for different characters such as (Mahpara, 2008, Ameen, 2008, Sharma and Chaudhary, 2009, and Akram et al., 2011).

Table (6): Estimates of SCA effects for studied characters in a $5 \times 5$ diallel cross of bread wheat.

\begin{tabular}{|l|c|c|c|c|c|c|}
\hline Crosses & $\begin{array}{c}\text { Days to } \\
\text { flowering }\end{array}$ & $\begin{array}{c}\text { Days to } \\
\text { maturity }\end{array}$ & $\begin{array}{c}\text { Plant height } \\
(\mathrm{cm})\end{array}$ & $\begin{array}{c}\text { Flag leaf } \\
\text { area }\left(\mathrm{cm}^{2}\right)\end{array}$ & $\begin{array}{c}\text { No. of } \\
\text { spikes/ } \\
\text { plant }\end{array}$ & $\begin{array}{c}\text { Spike } \\
\text { length }(\mathrm{cm})\end{array}$ \\
\hline $1 \times 2$ & -0.080 & 0.705 & 2.254 & 2.697 & 0.921 & 0.203 \\
$1 \times 3$ & -0.355 & 0.305 & 0.542 & 0.685 & 0.181 & -0.194 \\
$1 \times 4$ & -0.180 & 0.255 & 2.214 & 1.081 & 1.016 & 0.346 \\
$1 \times 5$ & 0.945 & 1.055 & 0.313 & 3.301 & 0.311 & 0.152 \\
$2 \times 3$ & 0.395 & 0.330 & 0.152 & 2.390 & 0.006 & 0.034 \\
$2 \times 4$ & -1.805 & -0.720 & 0.862 & 0.369 & 0.129 & -0.061 \\
$2 \times 5$ & 0.570 & -0.045 & 1.311 & 0.435 & -0.177 & 0.127 \\
$3 \times 4$ & 0.920 & 0.630 & -0.894 & -0.333 & 0.038 & -0.129 \\
$3 \times 5$ & -1.080 & -0.695 & 1.849 & 1.283 & 0.958 & 0.217 \\
$4 \times 5$ & -0.905 & -0.245 & 1.677 & 2.997 & 0.506 & 0.273 \\
\hline S.E. $\left(g_{i}-g_{j}^{\prime}\right)$ & 0.312 & 0.4254 & 0.599 & 0.740 & 0.282 & 0.088 \\
\hline
\end{tabular}

\begin{tabular}{|l|c|c|c|c|c|}
\hline Crosses & $\begin{array}{c}\text { No. of } \\
\text { grains/spike }\end{array}$ & $\begin{array}{c}\text { 1000-grain } \\
\text { weight }(\mathrm{g})\end{array}$ & $\begin{array}{c}\text { Biological } \\
\text { yield/plant }(\mathrm{g})\end{array}$ & $\begin{array}{c}\text { Grain } \\
\text { yield/plant }(\mathrm{g})\end{array}$ & $\begin{array}{c}\text { Harvest index } \\
(\%)\end{array}$ \\
\hline $1 \times 2$ & -0.234 & 2.978 & 9.190 & 4.022 & 0.165 \\
$1 \times 3$ & 0.323 & -0.377 & 0.422 & 0.638 & 0.459 \\
$1 \times 4$ & 2.498 & 0.998 & 8.744 & 4.721 & 0.929 \\
$1 \times 5$ & -3.264 & 2.588 & 6.117 & 0.870 & -1.582 \\
$2 \times 3$ & -0.862 & -0.850 & -2.078 & -1.211 & -0.502 \\
$2 \times 4$ & -3.074 & 0.677 & 2.894 & 1.073 & -0.195 \\
$2 \times 5$ & 3.589 & -1.001 & -0.883 & -0.072 & 0.281 \\
$3 \times 4$ & -0.204 & -1.564 & -1.586 & -1.550 & -1.021 \\
$3 \times 5$ & 1.621 & 1.554 & 7.425 & 4.165 & 1.116 \\
$4 \times 5$ & 3.859 & 0.039 & 4.710 & 3.034 & 1.108 \\
\hline S.E. $\left(g_{i}-g_{j}\right)$ & 0.878 & 0.583 & 2.229 & 1.084 & 0.373 \\
\hline
\end{tabular}

The data in Table $(7)$ showed that the cross $(3 \times 1)$ had a desirable reciprocal effects for days to flowering, days to maturity, plant height, grains per spike and harvest index, and a cross $(4 \times 1)$ for flag leaf area, spikes per plant, grain yield per plant and harvest index, then a cross $(4 \times 2)$ for days to maturity, plant height and harvest index and a cross $(5 \times 2)$ for plant height, 1000-grain weight and harvest index, then a cross $(5 \times 3)$ for grains per spike, 1000- grain weight and harvest index, then cross $(2 \times 1)$ for days to maturity and 1000 - grain weight, and $(3 \times 1)$ for spike length and grains per spike, and a cross $(4 \times 3)$ for harvest index, then a cross $(5 \times 1)$ for plant height. Reciprocal effects have obtained for various characters by other researchers when they used different genetic sources such as (Khan et al., 2007, Ameen, 2008, and Akram et al., 2011). 
Mesopotamia J. of Agric. Vol. (42) No. (1) 2014
ISSN: 2224-9796 (Online) ISSN: $1815-316 \mathrm{X}$ (Print)

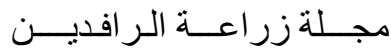

المجلد(42) العدد(1) 2014 اعلمن

Table (7): Estimates of reciprocal effects for studied characters in a $5 \times 5$ diallel cross of bread wheat.

\begin{tabular}{|l|c|c|c|c|c|c|}
\hline Reciprocals & $\begin{array}{c}\text { Days to } \\
\text { flowering }\end{array}$ & $\begin{array}{c}\text { Days to } \\
\text { maturity }\end{array}$ & $\begin{array}{c}\text { Plant } \\
\text { height }(\mathrm{cm})\end{array}$ & $\begin{array}{c}\text { Flag leaf } \\
\text { area }\left(\mathrm{cm}^{2}\right)\end{array}$ & $\begin{array}{c}\text { No. of } \\
\text { spikes/ } \\
\text { Plant }\end{array}$ & $\begin{array}{c}\text { Spike } \\
\text { length }(\mathrm{cm})\end{array}$ \\
\hline $2 \times 1$ & -0.250 & -0.625 & 0.706 & 0.628 & 0.075 & 0.061 \\
$3 \times 1$ & -1.000 & -1.375 & -1.337 & -0.424 & -0.262 & 0.037 \\
$4 \times 1$ & 0.125 & 0.000 & 0.500 & 1.411 & 0.300 & 0.045 \\
$5 \times 1$ & 0.125 & -0.250 & -1.175 & -2.495 & -1.475 & -0.374 \\
$3 \times 2$ & 0.250 & 0.000 & 0.275 & 0.500 & 0.138 & 0.114 \\
$4 \times 2$ & -0.250 & -1.125 & -2.525 & -1.485 & -0.212 & -0.263 \\
$5 \times 2$ & 0.000 & -0.250 & -1.050 & -2.765 & -0.212 & -0.346 \\
$4 \times 3$ & 1.000 & 0.125 & 0.863 & -3.503 & -0.450 & -0.144 \\
$5 \times 3$ & 0.125 & -0.250 & -0.119 & -2.321 & -0.250 & 0.003 \\
$5 \times 4$ & 0.250 & 1.125 & 0.813 & -0.319 & -0.075 & -0.211 \\
\hline S.E. $\left(r_{i j}-r_{i j}^{\prime}\right)$ & 0.293 & 0.388 & 0.547 & 0.675 & 0.258 & 0.081 \\
\hline
\end{tabular}

\begin{tabular}{|l|c|c|c|c|c|}
\hline Reciprocals & $\begin{array}{c}\text { No. of } \\
\text { grains/spike }\end{array}$ & $\begin{array}{c}\text { 1000-grain } \\
\text { weight }(\mathrm{g})\end{array}$ & $\begin{array}{c}\text { Biological } \\
\text { yield/plant }(\mathrm{g})\end{array}$ & $\begin{array}{c}\text { Grain } \\
\text { yield/plant }(\mathrm{g})\end{array}$ & $\begin{array}{c}\text { Harvest } \\
\text { index }(\%)\end{array}$ \\
\hline $2 \times 1$ & -0.237 & 0.953 & 0.475 & -0.072 & -0.249 \\
$3 \times 1$ & 1.337 & 0.105 & -1.913 & -0.110 & 0.871 \\
$4 \times 1$ & 0.500 & 0.530 & 1.163 & 1.556 & 1.100 \\
$5 \times 1$ & -3.525 & -0.428 & -11.925 & -5.191 & -0.469 \\
$3 \times 2$ & 1.600 & 0.260 & 1.150 & 0.602 & 0.285 \\
$4 \times 2$ & -2.225 & 0.047 & -4.525 & -1.444 & 0.715 \\
$5 \times 2$ & -3.550 & 1.788 & -1.162 & -0.139 & 0.531 \\
$4 \times 3$ & -2.813 & 0.520 & -3.350 & -1.103 & 0.538 \\
$5 \times 3$ & 0.925 & 1.270 & -1.975 & -0.166 & 0.875 \\
$5 \times 4$ & -1.125 & 0.105 & -1.088 & -0.689 & -0.203 \\
\hline S.E. $\left(r_{i j}-r_{i j}^{\prime}\right)$ & 0.802 & 0.532 & 2.035 & 0.990 & 0.340 \\
\hline
\end{tabular}

The evaluation of genetic and environmental variation $\left(\sigma_{\mathrm{A}}^{2}, \sigma_{\mathrm{D}}^{2}\right.$ and $\left.\sigma_{\mathrm{E}}^{2}\right)$ as presented in Table (8) for studied characters revealed that values for additive genetic variance were significant for all characters except for number of grains per spike and grain yield per plant, while values for dominance genetic and environment variances were significant for all the studied characters. Also the same table showed that the values of additive genetic variance were greater than dominance genetic variance for days to flowering, days to maturity, plant height, spike length, 1000-grain weight and harvest index, which depicts the importance of additive gene effect for controlling these characters, whereas the values of dominance genetic variance were greater than the additive genetic variance for flag leaf area, spikes per plant, grains per spike, biological yield and grain yield per plant, which indicates to the importance of dominant gene effect for controlling these characters. These results are in agreement with those previously reported by other researchers (Joshi, et al 2004, Hassan, 2004, Mahpara et al., 2008 and Ullah et al., 2010). 
Mesopotamia J. of Agric. Vol. (42) No. (1) 2014
ISSN: 2224-9796 (Online)

ISSN: $1815-316 \mathrm{X}$ (Print)

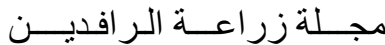

المجلد(42) العدد(1) 2014 اعلمن

Table (8): Estimates of genetic variation $\left(\sigma^{2}{ }_{A}, \sigma_{D}^{2}\right)$ and environmental variation $\sigma^{2}$ for studied characters.

\begin{tabular}{|l|c|c|c|c|c|c|}
\hline Variations & $\begin{array}{c}\text { Days to } \\
\text { flowering }\end{array}$ & $\begin{array}{c}\text { Days to } \\
\text { maturity }\end{array}$ & $\begin{array}{c}\text { Plant } \\
\text { height } \\
(\mathrm{cm})\end{array}$ & $\begin{array}{c}\text { Flag leaf } \\
\text { area } \\
\left(\mathrm{cm}^{2}\right)\end{array}$ & $\begin{array}{c}\text { No. of } \\
\text { spikes/ } \\
\text { plant }\end{array}$ & $\begin{array}{c}\text { Spike } \\
\text { length } \\
(\mathrm{cm})\end{array}$ \\
\hline Additive $\left(\sigma_{\mathrm{A}}^{2}\right)$ & $3.993^{* *}$ & $0.819^{* *}$ & $51.776^{* *}$ & $8.673^{* *}$ & $0.914^{* *}$ & $0.895^{* *}$ \\
& \pm 0.904 & \pm 0.219 & \pm 11.202 & \pm 2.836 & \pm 0.268 & \pm 0.192 \\
Dominance & $0.955^{* *}$ & $0.294^{*}$ & $7.038^{* *}$ & $14.448^{* *}$ & $0.990^{* *}$ & $0.093^{* *}$ \\
$\left(\sigma_{\mathrm{D}}^{2}\right)$ & \pm 0.208 & \pm 0.131 & \pm 1.361 & \pm 2.712 & \pm 0.204 & \pm 0.019 \\
& $0.428^{* *}$ & $0.753 * *$ & $1.496^{* *}$ & $2.279 * *$ & $0.332^{* *}$ & $0.033 * *$ \\
Environment & \pm 0.070 & \pm 0.124 & \pm 0.246 & \pm 0.375 & \pm 0.055 & \pm 0.005 \\
$\left(\sigma_{\mathrm{E}}^{2}\right)$ & & & & & & \\
\hline
\end{tabular}

\begin{tabular}{|l|c|c|c|c|c|}
\hline Variations & $\begin{array}{c}\text { No. of } \\
\text { grains/spike }\end{array}$ & $\begin{array}{c}\text { 1000-grain } \\
\text { weight }(\mathrm{g})\end{array}$ & $\begin{array}{c}\text { Biological } \\
\text { yield/plant } \\
(\mathrm{g})\end{array}$ & $\begin{array}{c}\text { Grain } \\
\text { yield/plant } \\
(\mathrm{g})\end{array}$ & $\begin{array}{c}\text { Harvest } \\
\text { index }(\%)\end{array}$ \\
\hline Additive $\left(\sigma_{\mathrm{A}}^{2}\right)$ & 1.742 & $11.700^{* *}$ & $51.237^{* *}$ & $1.614^{* *}$ & $1.551^{* *}$ \\
& \pm 0.995 & \pm 2.811 & \pm 17.688 & \pm 1.848 & \pm 0.386 \\
Dominance & $7.617 * *$ & $5.149 * *$ & $94.990^{* *}$ & $19.888^{* *}$ & $0.642^{* *}$ \\
$\left(\sigma_{\mathrm{D}}^{2}\right)$ & \pm 1.639 & \pm 1.029 & \pm 18.420 & \pm 3.915 & \pm 0.171 \\
& $3.212^{* *}$ & $1.416^{* *}$ & $20.702^{* *}$ & $4.900^{* *}$ & $0.578^{* *}$ \\
Environment & \pm 0.528 & \pm 0.233 & \pm 3.403 & \pm 0.806 & \pm 0.095 \\
$\left(\sigma_{\mathrm{E}}^{2}\right)$ & & & & \\
\hline & & & & & \\
\hline
\end{tabular}

Table (9) showed that the average degree of dominance (a) was more than one for flag leaf area, spikes per plant, grains per spike, biological yield/plant and grain yield/plant indicate that these characters are controlled by over dominance. These results are in agreement with previous results (Ameen, 2008, and Khattab et $a l ., 2010$ ); while it was less than one for days to flowering, days to maturity, plant height, spike length, 1000-grain weight and harvest index indicate that these characters are controlled by partial dominance. These results are in agreement with those previously reported by Hassan (2004) and Esmail (2007). The values of heritability in broad sense $\left(\mathrm{H}_{\text {b.s. }}^{2}\right)$ were high for all characters ranged between (\%74.447) for grains per spike and (\%97.519) for plant high except the days to maturity which was moderate (\%59.650). These findings correspond with the results published by some other authors (Ahmed et al., 2007, Ajmal et al., 2009, and Yadav et al., 2011). The estimated values of heritability in narrow-sense $\left(\mathrm{H}^{2}\right.$ n.s. varied between high for days to flowering, plant height, spike length, 1000-grain yield and harvest index and moderate for days to maturity, flag leaf area, spikes per plant and biological yield while they were low for grains per spike and grain yield per plant. These results agreed with those previously reported by Hassan, (2004), Eid, (2009), Khattab et al. (2010) and Erkul et al. (2010). The expected genetic advance (E.G.A) was between 0.553 for grain yield per plant and 11.734 for plant height. The expected genetic advance as a percent of mean was moderate for plant height, spike length and 1000-grain weight and it was moderate for spikes per plant, while it was low for the rest of the characters. These results are in agreement with those reported by Eid, (2009) and Bilgin et al. (2011). 
Mesopotamia J. of Agric. Vol. (42) No. (1) 2014
ISSN: 2224-9796 (Online) ISSN: $1815-316 \mathrm{X}$ (Print)
مجـلـة زر اعــة الر افديــن

المجلد(42) العدد(1) 2014 اعلمن

Table (9): Average degree of dominance and heritability in broad sense and narrow sense and expected genetic advance for studied characters.

\begin{tabular}{|l|c|c|c|c|c|c|}
\hline $\begin{array}{l}\text { Genetic } \\
\text { parameters }\end{array}$ & $\begin{array}{c}\text { Days to } \\
\text { flowering }\end{array}$ & $\begin{array}{c}\text { Days to } \\
\text { maturity }\end{array}$ & $\begin{array}{c}\text { Plant } \\
\text { height } \\
(\mathrm{cm})\end{array}$ & $\begin{array}{c}\text { Flag leaf } \\
\text { area } \\
\left(\mathrm{cm}^{2}\right)\end{array}$ & $\begin{array}{c}\text { No. of spikes/ } \\
\text { plant }\end{array}$ & $\begin{array}{c}\text { Spike } \\
\text { length } \\
(\mathrm{cm})\end{array}$ \\
\hline$(\overline{\mathrm{a}})$ & 0.692 & 0.847 & 0.521 & 1.825 & 1.472 & 0.457 \\
$\mathrm{H}_{\text {b.s. }}^{2}$ & 92.037 & 59.650 & 97.519 & 91.026 & 85.149 & 96.808 \\
$\mathrm{H}_{\text {n.s. }}^{\text {E.G.A. }}$ & 74.272 & 43.890 & 85.849 & 34.145 & 40.876 & 87.671 \\
E.G.A. & 3.031 & 1.055 & 11.734 & 3.029 & 1.076 & 1.559 \\
$(\%)$ & 2.305 & 0.610 & 10.982 & 5.802 & 9.020 & 11.484 \\
& & & & & & \\
\hline
\end{tabular}

\begin{tabular}{|l|c|c|c|c|c|}
\hline $\begin{array}{l}\text { Genetic } \\
\text { parameters }\end{array}$ & $\begin{array}{c}\text { No. of } \\
\text { grains/spike }\end{array}$ & $\begin{array}{c}\text { 1000-grain } \\
\text { weight }(\mathrm{g})\end{array}$ & $\begin{array}{c}\text { Biological } \\
\text { yield/plant } \\
(\mathrm{g})\end{array}$ & $\begin{array}{c}\text { Grain } \\
\text { yield/plant } \\
(\mathrm{g})\end{array}$ & $\begin{array}{c}\text { Harvest } \\
\text { index (\%) }\end{array}$ \\
\hline$(\overline{\mathrm{a}})$ & 2.957 & 0.938 & 1.926 & 4.964 & 0.910 \\
$\mathrm{H}^{2}{ }_{\text {b.s. }}$ & 74.447 & 92.250 & 87.599 & 81.442 & 79.135 \\
$\mathrm{H}^{2}$ n.s. & 13.857 & 64.057 & 30.694 & 6.114 & 55.956 \\
E.G.A. & 0.865 & 4.818 & 6.980 & 0.553 & 1.640 \\
E.G.A. (\%) & 1.228 & 10.256 & 8.836 & 1.625 & 3.801 \\
\hline
\end{tabular}

The estimates of heterosis over mid parents of $\mathrm{F} 1$ hybrids and reciprocals for all the studied characters are presented in Table (10) revealed that the best crosses which showed the maximum heterosis in a desirable direction for grain yield per plant were $(1 \times 4)$ and $(5 \times 1)$ and they recorded 15.043 and $15.185 \mathrm{~g} / \mathrm{plant}$, these hybrids also showed the desirable heterosis in other characters like flag leaf area, spikes per plant, spike length, 1000-grain weight, biological yield per plant and harvest index. In addition to desirable SCA by cross $(1 \times 4)$ for flag leaf area, spikes per plant, spike length, number of grains per spike, 1000-grain weight, biological yield, grain yield and harvest index. Based on these results, it is possible to use heterosis estimates for evaluation of dominance gene action in hybrids. The appearance of the heterosis over mid parents for any characters indicate that the dominance gene action controlled it, while the hybrid which doesn't show significant heterosis indicated that the additive gene action controlled it (Ansari, 2002) and agrees that dominance hypothesis is an acceptable explanation of heterosis. Based on the results of this study showed that $\% 67$ of heterosis were for characters that controlled by dominant gene action more than additive gene action for their inheritance, while $\% 42.8$ of heterosis characters that controlled by additive gene action, and this is coming from the fact that both of heterosis and specific combining ability are originated from the dominance gene effects (Shamsuddin, 1985). These results are in agreement with those reported by other researchers (Kundan et al., 2010, Khattab et al., 2010, Akbar et al., 2010 and Ilker et al., 2010). 
Mesopotamia J. of Agric.

Vol. (42) No. (1) 2014
ISSN: 2224-9796 (Online) ISSN: $1815-316 \mathrm{X}$ (Print)
مجـلـة زر اعــة الر افديــن

المجلد(42) العدد(1) 2014 اعلمن

Table (10): Heterosis estimates of studied characters in bread wheat of mid parent.

\begin{tabular}{|l|c|c|c|c|c|c|}
\hline Crosses & $\begin{array}{c}\text { Days to } \\
\text { flowering }\end{array}$ & $\begin{array}{c}\text { Days to } \\
\text { maturity }\end{array}$ & $\begin{array}{c}\text { Plant } \\
\text { height }(\mathrm{cm})\end{array}$ & $\begin{array}{c}\text { Flag leaf } \\
\text { area }\left(\mathrm{cm}^{2}\right)\end{array}$ & $\begin{array}{c}\text { No. of spikes/ } \\
\text { plant }\end{array}$ & $\begin{array}{c}\text { Spike } \\
\text { length }(\mathrm{cm})\end{array}$ \\
\hline $1 \times 2$ & -0.625 & 1.375 & $7.911^{* *}$ & $10.153^{* *}$ & $2.651^{* *}$ & $0.670^{* *}$ \\
$1 \times 3$ & -1.250 & 0.375 & 2.690 & $6.155^{* *}$ & $1.725^{*}$ & 0.061 \\
$1 \times 4$ & -0.875 & 1.375 & $7.300^{* *}$ & $8.431^{* *}$ & $3.375^{* *}$ & $0.860^{* *}$ \\
$1 \times 5$ & 1.000 & 2.000 & $4.374^{* *}$ & 8.696 & 0.850 & 0.416 \\
$2 \times 3$ & 0.125 & 0.750 & $3.541^{*}$ & $7.848^{* *}$ & 1.175 & 0.264 \\
$2 \times 4$ & $-3.500^{* *}$ & -1.750 & 2.556 & $3.886^{*}$ & 1.200 & 0.042 \\
$2 \times 5$ & -0.125 & -0.125 & $5.125^{* *}$ & $4.624^{*}$ & 0.850 & 0.316 \\
$3 \times 4$ & 0.875 & 1.000 & 2.723 & 0.234 & 1.025 & -0.094 \\
$3 \times 5$ & -1.250 & -0.625 & $5.129^{* *}$ & $4.981^{* *}$ & $2.100^{* *}$ & $0.568^{*}$ \\
$4 \times 5$ & $-1.875^{* *}$ & 0.875 & $6.994^{* *}$ & $8.743^{* *}$ & $2.075^{* *}$ & $0.661^{* *}$ \\
$5 \times 4$ & $-2.375^{* *}$ & -1.375 & $5.369^{* *}$ & $9.380^{* *}$ & $2.225^{* *}$ & $1.084^{* *}$ \\
$5 \times 3$ & -1.500 & -0.125 & $5.366^{* *}$ & $9.624^{* *}$ & $2.600^{* *}$ & $0.563^{*}$ \\
$5 \times 2$ & -0.125 & 0.375 & $7.225^{* *}$ & $10.154^{* *}$ & 1.275 & $1.009^{* *}$ \\
$5 \times 1$ & 0.750 & $2.500^{*}$ & $6.724^{* *}$ & $13.686^{* *}$ & $3.800^{* *}$ & $1.164^{* *}$ \\
$4 \times 3$ & -1.125 & 0.750 & 0.997 & $7.239^{* *}$ & $1.925^{* *}$ & 0.194 \\
$4 \times 2$ & $-3.000^{* *}$ & 0.500 & $7.606^{* *}$ & $6.856^{* *}$ & $1.625^{*}$ & $0.567 *$ \\
$4 \times 1$ & -1.125 & 1.375 & $6.305^{* *}$ & $5.609^{* *}$ & $2.775^{* *}$ & $0.770^{* *}$ \\
$3 \times 2$ & -0.375 & 0.750 & 2.991 & $6.848^{* *}$ & 0.900 & 0.036 \\
$3 \times 1$ & 0.750 & $3.125 * *$ & $5.365^{* *}$ & $7.003^{* *}$ & $2.250^{* *}$ & -0.014 \\
$2 \times 1$ & -0.125 & 2.625 & $6.499^{* *}$ & $8.897^{* *}$ & $2.500^{* *}$ & $0.547^{*}$ \\
\hline
\end{tabular}

\begin{tabular}{|l|c|c|c|c|c|}
\hline Crosses & $\begin{array}{c}\text { No. of } \\
\text { grains/spike }\end{array}$ & $\begin{array}{c}\text { 1000-grain } \\
\text { weight }(\mathrm{g})\end{array}$ & $\begin{array}{c}\text { Biological } \\
\text { yield/plant }(\mathrm{g})\end{array}$ & $\begin{array}{c}\text { Grain } \\
\text { yield/plant }(\mathrm{g})\end{array}$ & $\begin{array}{c}\text { Harvest index } \\
(\%)\end{array}$ \\
\hline $1 \times 2$ & -1.100 & $7.925^{* *}$ & $26.463^{* *}$ & $10.981^{* *}$ & -0.223 \\
$1 \times 3$ & 1.762 & 2.203 & $12.838^{*}$ & $6.675^{*}$ & 1.343 \\
$1 \times 4$ & 4.200 & $4.695^{* *}$ & $29.525^{* *}$ & $15.043^{* *}$ & $2.426^{*}$ \\
$1 \times 5$ & -4.225 & $6.843^{* *}$ & $15.113^{* *}$ & 4.802 & -1.603 \\
$2 \times 3$ & 0.888 & -0.308 & 5.725 & 2.319 & -0.315 \\
$2 \times 4$ & -4.050 & 1.700 & 10.313 & 5.174 & 0.806 \\
$2 \times 5$ & 2.650 & $3.278^{*}$ & $11.200^{*}$ & $5.694^{*}$ & 1.150 \\
$3 \times 4$ & -1.037 & -1.588 & 4.537 & 2.008 & -0.046 \\
$3 \times 5$ & $5.888^{* *}$ & $3.795^{*}$ & $16.225^{* *}$ & $9.018^{* *}$ & $2.480^{* *}$ \\
$4 \times 5$ & $7.175^{* *}$ & 1.808 & $19.688^{* *}$ & $9.983^{* *}$ & 1.779 \\
$5 \times 4$ & $9.425^{* *}$ & 1.598 & $21.863^{* *}$ & $11.360^{* *}$ & $2.184 *$ \\
$5 \times 3$ & 4.037 & 1.255 & $20.175^{* *}$ & $9.350^{* *}$ & 0.730 \\
$5 \times 2$ & $9.750^{* *}$ & -0.298 & $13.525^{* *}$ & $5.971^{*}$ & 0.087 \\
$5 \times 1$ & 2.825 & $7.698^{* *}$ & $38.963^{* *}$ & $15.185^{* *}$ & -0.665 \\
$4 \times 3$ & $4.588^{* *}$ & -2.628 & $11.238^{*}$ & 4.213 & -1.121 \\
$4 \times 2$ & 0.400 & 1.605 & $19.363^{* *}$ & $8.061^{* *}$ & -0.624 \\
$4 \times 1$ & 3.200 & $3.635^{*}$ & $27.200^{* *}$ & $11.930^{* *}$ & 0.226 \\
$3 \times 2$ & -2.213 & -0.828 & 3.425 & 1.114 & -0.885 \\
$3 \times 1$ & -0.912 & 1.993 & $16.663^{* *}$ & $6.895^{*}$ & -0.400 \\
$2 \times 1$ & -0.625 & $6.020^{* *}$ & $25.513^{* *}$ & $11.126^{* *}$ & 0.275 \\
\hline
\end{tabular}

* Significant $(\mathrm{P}=0.05), \quad * *$ Significant $(\mathrm{P}=0.01)$

Genetic correlation coefficients between studied characters are shown in Table (11). There were significant and positive genetic correlation between grain yield and most of its components and between themselves, thus selection for any of these characters lead to improve other characters including grain yield. Similar 
Mesopotamia J. of Agric.

Vol. (42) No. (1) 2014

results have been reported by Ajmal et al. (2009) and Bilgin et al. (2011). The results of this study revealed that parents (1), (2) and (5) had the possibility to use in crossing programs because they have showed desirable GCA effects for most of the studied characters and obtaining some crosses like $(3 \times 5),(5 \times 4),(4 \times 5)$ and $(1 \times 4)$, which they had high desirable heterosis for most of the characters and could used in the future breeding programs.

Table (11): Genetic correlation coefficients between studied characters.

\begin{tabular}{|c|c|c|c|c|c|c|c|c|c|c|}
\hline & $\begin{array}{l}\text { Harvest } \\
\text { index }\end{array}$ & $\begin{array}{c}\text { Grain } \\
\text { yield/plant }\end{array}$ & $\begin{array}{l}\text { Biological } \\
\text { yield/plant }\end{array}$ & $\begin{array}{c}1000- \\
\text { grain } \\
\text { weight }\end{array}$ & $\begin{array}{l}\text { No. of } \\
\text { grains/ } \\
\text { spike }\end{array}$ & $\begin{array}{l}\text { Spike } \\
\text { length }\end{array}$ & $\begin{array}{c}\text { No. of } \\
\text { spikes/plant }\end{array}$ & $\begin{array}{l}\text { Flag } \\
\text { leaf } \\
\text { area } \\
\end{array}$ & $\begin{array}{c}\text { Plant } \\
\text { height }\end{array}$ & $\begin{array}{l}\text { Days to } \\
\text { maturity }\end{array}$ \\
\hline $\begin{array}{c}\text { Days to } \\
\text { flowering }\end{array}$ & $.609 * *$ & $-.609 * *$ & $-.675^{* *}$ & -.226 & .239 & $-.407 *$ & $-.721 * *$ & $.517^{* * *}$ & $-.554 * *$ & .030 \\
\hline $\begin{array}{l}\text { Days to } \\
\text { maturity }\end{array}$ & $-.417 *$ & $.514 * *$ & $.576^{* *}$ & $.860 * *$ & $-.602 * *$ & $.797 * *$ & .378 & $.677 * *$ & $.762 * *$ & \\
\hline $\begin{array}{c}\text { Plant } \\
\text { height }\end{array}$ & $-.545 * *$ & $.786^{* *}$ & $.830 * *$ & $.624 * *$ & $-.408^{*}$ & $.971 * *$ & $.761 * *$ & $.862 * *$ & & \\
\hline $\begin{array}{c}\text { Flag leaf } \\
\text { area }\end{array}$ & $-.467 *$ & $.921 * *$ & $.940 * *$ & $.551 * *$ & -.127 & .820 *** & $.910^{* * *}$ & & & \\
\hline $\begin{array}{c}\text { No. of } \\
\text { spikes/plant }\end{array}$ & $-.496^{*}$ & $.883 * *$ & $.912 * *$ & .338 & -.151 & $.609 * *$ & & & & \\
\hline $\begin{array}{l}\text { Spike } \\
\text { length }\end{array}$ & $-.483^{*}$ & $.707 * *$ & $.746^{* *}$ & $.691 * *$ & -.345 & & & & & \\
\hline $\begin{array}{l}\text { No. of } \\
\text { grains/ } \\
\text { spike }\end{array}$ & $.719 * *$ & -.065 & -.231 & -.393 & & & & & & \\
\hline $\begin{array}{c}\text { 1000-grain } \\
\text { weight }\end{array}$ & -.318 & $.698^{* *}$ & $.709 * *$ & & & & & & & \\
\hline $\begin{array}{c}\text { Biological } \\
\text { yield/plant }\end{array}$ & $-.510 * *$ & $.973^{* *}$ & & & & & & & & \\
\hline $\begin{array}{c}\text { Grain } \\
\text { yield/plant }\end{array}$ & -.302 & & & & & & & & & \\
\hline
\end{tabular}

\author{
تحليل القرة على الائتلاف والفعل الجيني وقوة الهجين \\ للتهجين التبادلي الكامل في حنطة الخبز

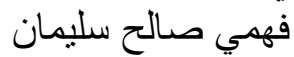 \\ مركز البحوث الزر اعية/ كويان \\ اسماعيل حسين علي \\ كلية الزر اعة/ جامعة صلاّح الدين علين
}

\title{
الخلاصة
}

تضمنت هذه التجربة إجر اء تهجين تبادلي كامل بين خمسة تراكيب وراثية من حنطة الخبز في

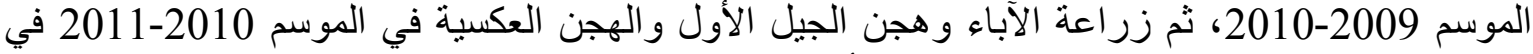
محطة التجارب الزراعية في كوية/محافظة أربيل تحت الظروف الإيمية باستخدام تصميم القطاعات العشوائية الكاملة بأربعة مكررات، ودرست بعض صفات النمو والحاصل الحاتل ومكوناته. أجري التحليل

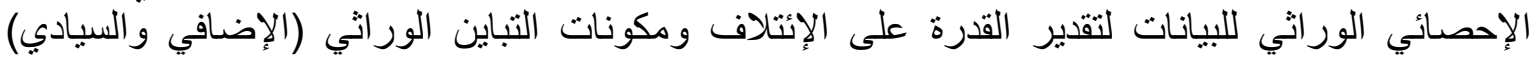

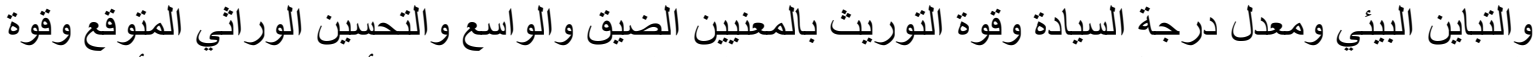

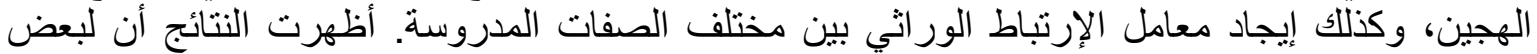

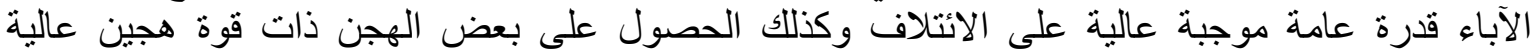

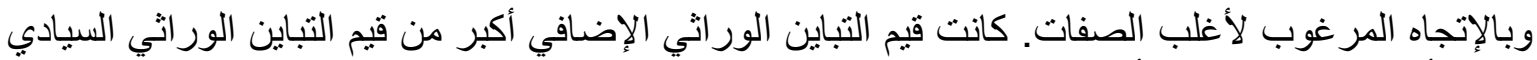

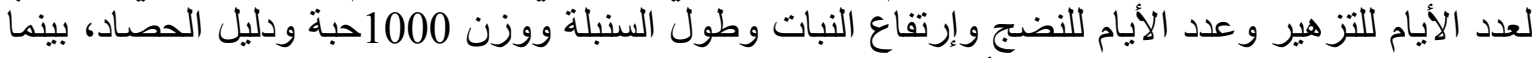

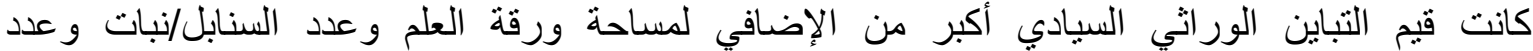

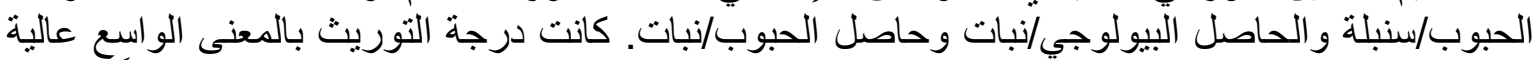

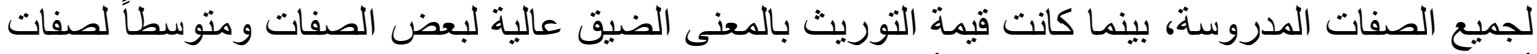

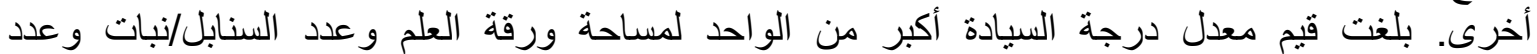

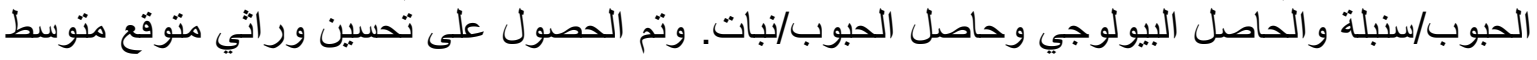


Mesopotamia J. of Agric.

Vol. (42) No. (1) 2014
ISSN: 2224-9796 (Online)

ISSN: $1815-316$ X (Print)

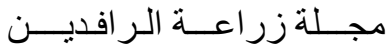

المجلد(42) العدد(1) 2014 اعلمن

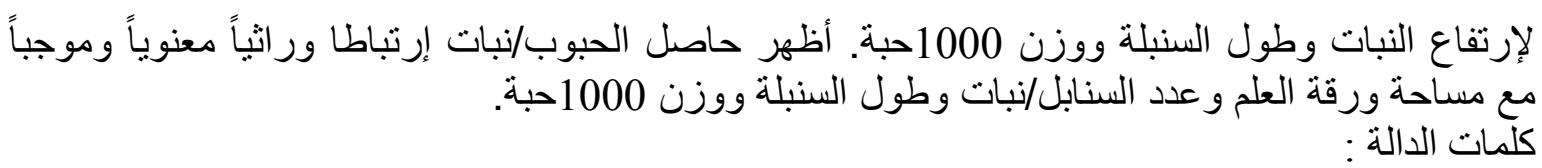

تاريخ تسلم البحث : 2011/12/5 وقبوله 2012/9/10

\section{REFERENCES}

Adary, A. H. M. (1987). Principles of Genetics. $2^{\text {nd }}$ edition. Mosul Univ. Press. (In Arabic).

Agarwal, V. and Z. Ahmad (1982). Heritability and genetic advance in triticale. Indian Journal of Agriculture Research, 16:19-23.

Ahmed, N., M. A. Chowdhry, I. Khaliq, and M. Maekawa (2007). The inheritance of yield and yield components of five wheat hybrid populations under drought conditions. Indonesian Journal of Agricultural Science, 8(2):53-59.

Ajmal, S. U., N. Zakir, and M. Y. Mujahid (2009). Estimation of genetic parameters and character association in wheat. Journal of Agriculture and Biological Science, 1(1):15-18.

Akbar, M., J. Anwar, M. Hussain, M. M. Iqbal and W. Sabir (2010). Heterosis and heterobeltiosis for grain yield improvement in bread wheat. Journal of Agriculture Research, 48(1): 15 - 23.

Akram, Z., S. Ajmal, K. S. Khan, R. Qureshi and M. Zubair (2011). Combining ability estimates of some yield and quality related characters in spring wheat (Triticum aestivum L.). Pakistan Journal of Botany, 43(1): 221-231.

Akram, Z., S. U. Ajmal, G. Shabbir, M. Munir and N. M. Cheema (2009). Inheritance mechanism of some yield components in bread wheat. Pakistan Journal of Agriculture Research, 22(1-2): 1 - 8 .

Ali, A. A. (1999). Heterosis and Gene Action in Corn (Zea mays L.). Ph. D. Thesis, College of Agriculture and Forestry, Mosul University. (In Arabic).

Ameen, T. N. H. (2008). Full Diallel Crosses in Durum Wheat (Triticum durum). M. Sc. Thesis, College of Agriculture, University of Sulaimani (Iraq).

Ansari, B. A. (2002). Diallel Analysis of Yield and Yield Components in Triticum aestivum L. Ph. D. Thesis, Faculty of Crop Production, Sindh Agriculture University, Tando Jum.

Bilgin, O., K. Z. Korkut, I. Başer, O. Dağlioğlu, I. Öztürk, T. Kahraman and A. Balkan (2011). Genetic variation and inter-relationship of some morphophysiological characters in durum wheat (Triticum durum L. desf.). Pakistan Journal of Botany, 43(1): 253-260.

Cheema, N., M., M. A. Mian, M. Ihsan, G. Rabbani and A. Mahmood (2006). Studies on variability and some genetic parameters in spring wheat. Pakistan Journal of Agriculture Science, 43(1-2): 32 - 35.

Eid, M. H. (2009). Estimation of heritability and genetic advance of yield characters in wheat (Triticum aestivum L.) under drought condition. International Journal of Genetics and Molecular Biology, 1(7): 115-120.

Erkul, A., A. Unay and C. Konak (2010). Inheritance of yield and yield components in a bread wheat (Triticum aestivum L.) cross. Turkish Journal of Field Crops, 15(2):137-140. 
Esmail,R. M. (2007). Detection of genetic components through triple test crosses and line $\times$ tester analysis in bread wheat. World Journal of Agriculture Science, 3(2): 184-190.

Griffing, B. (1956). Concept of general and specific combining ablility in relation to diallel crossing systems. Australian Journal of Biological Science, 9:463493.

Hassan, G. (2004). Diallel Analysis of Important Parameters in Wheat (Triticum aestivum L.) under Irrigated and Rainfed Conditions. Ph. D. Thesis, Faculty of Crop Production Sciences, N.W.F.P Agricultural University, Peshawar, Pakistan.

Hayman, B. I. (1954). The theory and analysis of diallel crosses. Genetics, 39: 789809.

İlker, E. F., A. Tonk and M. Tosun (2010). Heterosis for yield and its components in bread wheat crosses among powdery mildew resistant and susceptible genotypes. Pakistan Journal of Botany, 42(1): 513 - 522.

Jinks , J. L. and B. I. Hayman (1953). The analysis of diallel crosses. Maize Genetic Newsletter, 27: 48-54.

Joshi, S. K., S. N. Sharma, D.L. Singhania and R.S. Sain (2004). Combining ability in the F1 and F2 generations of diallel cross in hexaploid wheat (Triticum aestivum L. em. Thell). Hereditas, 141: $115-121$.

Kashif, M. and I. Khaliq (2003). Determination of general and specific combining ability effects in a diallel cross of spring wheat. Pakistan Journal of Biological Science, 6(18): 1616 - 1620.

Kempthorene, O. 1969. An Introduction to Genetic Statistics. Ames, Iowa State University Press (USA).

Khan, M.A., N. Ahmad, M. Akbar, A. Rehman and M. M. Iqbal (2007). Combining ability analysis in wheat. Pakistan Journal of Agriculture Science, 44(1): 1 -5 .

Khattab, S. A. M., R. M. Esmail and A. M. F. AL-Ansary (2010). Genetical analysis of some quantitative characters in bread wheat (Triticum aestivum L.). New York Science Journal, 3(11): 152 - 157.

Kundan, K. J., P. Pandey, S. Marker and P. J. Anurag (2010). Heterosis studies for improvement in yield potential of wheat (Triticum aestivum L.). Advanced in Agriculture and Botanics-International Journal of the Bioflux Society, 2 (3):273-278.

Liu, Y. (2005). Diallel and Stability Analysis of Kenaf (Hibiscus cannabinus L.) in South Africa. M. Sc. Thesis. Faculty of Natural and Agricultural Sciences, Plant Breeding University of the Free State Bloemfontein (South Africa).

Mahpara, Sh. (2008). Biometrical Analysis of Important Plant Attributes in Spring Wheat. Ph. D. Thesis, Faculty of Agriculture, University of agriculture, Faisalabad (Pakistan).

Mahpara, SH., Z. Ali and M. Ahsan (2008). Combining ability analysis for yield and yield related characters among wheat varieties and their F1 hybrids. International Journal of Agriculture and Biology, 10(6): 599-604.

Shamsuddin, A. K. M. (1985). Genetic diversity in relation to heterosis and combining ability in spring wheat. Theoretical Applied Genetics, (70):306308. 
Sharma, S. and H. K. Chaudhary (2009). Combining ability and gene action studies for yield-contributing characters in crosses involving winter and spring wheat genotypes. Acta Agronomica Hungarica, 57(4): 417- 423.

Singh, R. K. and B. D. Chaudhary (1985). Biometrical Methods in Quantitative Genetic Analysis. Kalyani Publisher, Ludhiana( India).

Sprague, G. F. and L. A. Tatum, (1942). General versus specific combining ability in single crosses of corn. Journal of American Society and Agronomy, 34: 923-932.

Ullah, S., A. S. Khan, A. Raza and S. Sadique (2010). Gene action analysis of yield and yield related characters in spring wheat (Triticum aestivum). International Journal of Agriculture and Biology, 12(1): 125-128.

Walter, A. B. (1975). Manual of Quantitative Genetics. 3rd ed. Washington State University (USA).

Yadav, A. K. and A. Sirohi (2011). Combining ability for grain yield and other related characters in bread wheat (Triticum aestivum L.). Electronic Journal of Plant Breeding, 2(3):303-309.

Yadav, A. K., R. K. Maan, S. Kumar, P. Kumar (2011). Variability, heritability and genetic advance for quantitative characters in hexaploid wheat (Triticum aestivum L.). Electronic Journal of Plant Breeding, 2(3); 405-408. 